\title{
Alfven Soliton and Intermediate Shock in the Solar Wind
}

\author{
Song Liting ${ }^{1, *}$ and Xiao Chijie ${ }^{1}$ \\ (Manuscript received 1 October 2000, in final form 6 November 2000)
}

\begin{abstract}
We show in this paper that intermediate shocks are formed from kinetic Alfven waves through wave form continuous steepening. From the action of nonlinear interaction and dispersion Alfven solitons are formed and then evolve into shocks. We calculated the traveling stationary structures directly from nonlinear autonomous equations which we had derived. These traveling stationary structures correspond to intermediate shock, switch-on shock or Alfven solitary waves. Another kind of rare discontinuity was discovered in the calculation from the rarefying solitary waves. The solitary wave series remain on the front of the shocks because of no strong dissipation appears in the equation. When we added some anomalous dissipation terms to the equations, intermediate shocks with increased in entropy appeared.
\end{abstract}

(Key words: Kinetic Alfven waves, Intermediate shock, Alfven soliton)

\section{INTRODUCTION}

Is there any intermediate shock in the solar wind? How is intermediate shock produced? These are problems discussed by space scientists recently. Usually, magnetohydrodynamic (MHD) shocks are investigated through Rankine-Hugoniot jump relations. MHD shocks which satisfy the principle of discontinuity and entropy increase can be divided into three categories according to MHD wave modes: fast shock, slow shock and intermediate shock. But intermediate shock is usually referred to as non-evolutionary or unstable, and has been thought nonphysical reality (Jeffrey and Taniuti 1964; Anderson 1965). Wu (Wu 1987, 1988, 1990) has presented numerical solutions to dissipative MHD equations showing that MHD-intermediate shocks can be formed from continuous wave form steepening, and changing the parameters of dissipation can make intermediate shock stable through its structure layer variation. The existence and formation of intermediate shock has become an important subject for discussion. Some people pointed out, using numerical simulations and analyses, that in the shocks' evolu-

\footnotetext{
${ }^{1}$ Department of geophysics, Peking University, Beijing, China

${ }^{*}$ Corresponding author address: Prof. Song Liting, Department of geophysics, Peking University, Beijing 18071, China; E-mail: xielun@pku.edu.cn
} 
tion process the existence of intermediate shock is absolutely necessary (Kennel et al. 1989; Steiwolfson and Hundhauson 1990; Hu 1994). Furthermore, the existence of intermediate shocks is a necessary condition for a hydrid shock formed by linking up different types of MHD shock (Hu 1994).

Up to now, most interplanetary shocks measured have been affirmed to be fast shocks and some to be slow shocks (Chao 1974; Whang 1982, 1986a; Richter 1985, 1987), only one intermediate shock measured in the solar wind was reported (Chao 1993). Though intermediate shocks are thought to exist, it is still not clear, in theory how they are formed in a collisionless plasma. Corresponding to MHD wave modes, intermediate shock should be formed through Alfven wave evolution. But the Alfven wave is a kind of transverse wave propagating along the magnetic field and has no nonlinear interaction and dissipation in the theory. Some people offer nonlinear term $(\vec{V} \cdot \nabla) \vec{V}$ in the oblique propagation of the Alfven wave. But, group velocity of the Alfven wave, i.e., the wave energy still persists in the propagation along the magnetic field. Below we show that intermediate shock is evolved from the kinetic Alfven wave instead of from the MHD Alfven wave. The kinetic Alfven wave was first introduced in space physics by Hasegawa (Hasegawa 1976). It is weak dispersion, the dispersion relation is expressed approximately as:

$$
\omega^{2}=k_{l /}^{2} V_{A}^{2}\left(1+k_{\perp}^{2} \rho_{i}^{2}\right)
$$

Where $k_{\| /}$and $k_{\perp}$ are the wave vector component parallel and perpendicular to the magnetic field respectively, and $k_{\perp} \gg k_{/ /}$stand for the kinetic Alfven wave. $\rho_{i}$ is the ion gyroradius. In the solar wind it seems reasonable for $k_{\perp}^{2} \rho_{i}^{2}<<1$, although $k_{\perp} \gg k_{l /}$ for the kinetic Alfven wave energy is propagating along the magnetic field:

$$
\begin{gathered}
V_{g / l}=\frac{\partial \omega}{\partial k_{\| l}}=V_{A}\left(1+\frac{1}{2} k_{\perp}^{2} \rho_{i}^{2}\right) \geq V_{A}, \\
V_{g \perp}=\frac{\partial \omega}{\partial k_{\perp}}=V_{A} \frac{k_{l l}}{k_{\perp}}\left(k_{\perp}^{2} \rho_{i}^{2}\right)<<V_{A} .
\end{gathered}
$$

As there are longitudinal disturbed velocity and electric field $E_{/ /}$in the kinetic alfven wave the governing equations are nonlinear. From the action of the dispersion, the nonlinear kinetic Alfven wave first evolves into Alfven solitons, then into intermediate shock by the dissipation effect. The kinetic Alfven wave is quite different from the MHD Alfven wave. Besides having the dispersion and Landau dissipation, the kinetic Alfven wave is a longitudinal- transverse coupling mode, i.e., the Alfven wave and the ion acoustic wave coupling mode. Its transverse velocity and electric field in the wave yield elliptic polarization, so it easily evolves into kinklike solitons or eddies. In general, a shock must be a traveling. stationary structure that can be numerically calculated to be found. We can directly calculate this traveling stationary structure. We will show by numerical analysis that the dispersive, nonlinear kinetic Alfven wave can evolve into intermediate shocks, Alfven solitons and magnetic kink along with the different parameters in the solar wind. 


\section{GOVERNING EQUATIONS}

The solar wind is low $\beta$ plasma, $\frac{m_{e}}{m_{i}} \ll \beta<<1$ (where $\beta$ is the ratio of thermal pressure to magnetic pressure in the plasma). The inertial term of electron in the two-fluid equations can be omitted (Lysak and Lotko 1996). Suppose the background magnetic field is $B_{0}=B_{0} \hat{z}$, and we select the transverse disturbed electric field in the direction $\hat{x}$. Because the drift velocities of electron and ion driven by the electric field are the same in direction $\hat{y}$, we can discuss all variables in the $x-z$ plane. The two-dimensional equations of motion in the solar wind frame and Maxwell equations can be written in the form

$$
\begin{gathered}
-\frac{e}{m_{e}} E_{z}-\frac{C_{e}^{2}}{n} \frac{\partial n}{\partial z}=0, \\
\frac{\partial n}{\partial t}+\frac{\partial}{\partial x}\left(n v_{i x}\right)+\frac{\partial}{\partial z}\left(n v_{i z}\right)=0, \\
v_{i x}=\frac{c}{B_{0} \Omega_{i}} \frac{\partial E_{X}}{\partial t}, \\
\frac{\partial v_{i z}}{\partial t}+v_{i x} \frac{\partial v_{i z}}{\partial x}+v_{i z} \frac{\partial v_{i z}}{\partial z}=\frac{e}{m_{i}} E_{z}+\frac{e}{c m_{i}} v_{i x} B_{y}-\frac{c_{i}^{2}}{n} \frac{\partial n}{\partial z}, \\
\frac{\partial E_{x}}{\partial z}-\frac{\partial E_{z}}{\partial x}=-\frac{1}{c} \frac{\partial B_{y}}{\partial t}, \\
\frac{\partial B_{y}}{\partial z}=-\frac{4 \pi}{c} e n v_{i x} .
\end{gathered}
$$

Here we have used the quasi-neutrality condition $n=n_{i}=n_{e}$, where $c_{i}, c_{e}$ are the thermal speed of electrons and ions. As $m_{e} \ll m_{i}$ the electron polarization has been ignored. The displacement current in the Maxwell equations is omitted for $\omega<<\Omega_{i}$. Here we use Gauss units system, $\mathrm{c}$ is the speed of light. Canceling $v_{i x}$ in the equations above, we can normalize the equations as

$$
\begin{gathered}
\frac{1}{N} \frac{\partial N}{\partial Z}=-\frac{V_{A}^{2}}{C_{s}^{2}} E_{z} \\
\frac{\partial N}{\partial \tau}+\frac{\partial}{\partial Z}(N U)+\frac{\partial}{\partial X}\left(N \frac{\partial E_{X}}{\partial \tau}\right)=0 \\
\frac{\partial U}{\partial \tau}+U \frac{\partial U}{\partial Z}+\frac{\partial E_{x}}{\partial \tau} \frac{\partial U}{\partial x}=\left(1+\frac{T_{i}}{T_{e}}\right) E_{z}+B \frac{\partial E_{X}}{\partial \tau},
\end{gathered}
$$




$$
\begin{gathered}
\frac{\partial E_{X}}{\partial Z}+\frac{\partial E_{Z}}{\partial X}=-\frac{\partial B}{\partial \tau}, \\
\frac{\partial B}{\partial Z}=-N \frac{\partial E_{X}}{\partial \tau},
\end{gathered}
$$

where

$$
\begin{aligned}
& N=\frac{n}{n_{0}}, \mathrm{~B}=\frac{B_{y}}{B_{0}}, U=\frac{v_{i z}}{V_{A}}, E_{z}=\frac{E_{z} c}{B_{0} V_{A}}, E_{X}=\frac{E_{x} c}{B_{0} V_{A}}, X=x \frac{\Omega_{i}}{V_{A}}, Z=z \frac{\Omega_{i}}{V_{A}}, \tau=t \Omega_{i} ; \\
& C_{s}=\sqrt{\frac{k T_{e}}{m_{i}}} \text { is the ion acoustic speed normalized by } V_{A}\left(V_{A}=\frac{B_{0}}{\sqrt{4 \pi n_{i} n_{0}}}\right) \text {, which is the Alfven }
\end{aligned}
$$

velocity; $n_{0}$ is the density of background plasma. The Equations (1)-(5) can be transfered to the travelling wave phase:

$$
\begin{gathered}
\xi=\left(\alpha X+\gamma Z-M_{A} \tau\right) / M_{i}, \\
\frac{\partial}{\partial X}=\frac{\alpha}{M_{i}} \frac{\partial}{\partial \xi}, \frac{\partial}{\partial Z}=\frac{\gamma}{M_{i}} \frac{\partial}{\partial \xi}, \frac{\partial}{\partial \tau}=\frac{M_{A}}{M_{i}} \frac{\partial}{\partial \xi},
\end{gathered}
$$

where $\alpha=\frac{k_{x}}{k}, \gamma=\frac{k_{z}}{k}, M_{i}=\frac{\Omega_{i}}{k V_{A}}$, and $M_{A}=\frac{\omega}{k V_{A}}$, which is Alfven Mach number.

Equation (1) becomes:

$$
E_{z}=-\frac{\gamma}{M_{i}} \frac{C_{s}^{2}}{V_{A}^{2}} \frac{1}{N} \frac{\partial N}{\partial \xi}
$$

Integrating equation (2) with boundary condition $\xi \rightarrow-\infty, N=1, U=V_{1}$, yields

$$
U=\frac{M_{A}}{\gamma}+\frac{V_{1}-M_{A} / \gamma}{N}+\frac{\alpha M_{A}}{\gamma M_{i}} \frac{\partial E_{x}}{\partial \xi} .
$$

Substituting $E_{2}$ and $U$ into equations (3) and (4), with tedious derivations we obtain the normal nonlinear autonomous equations on $\xi$

$$
\begin{gathered}
\frac{\partial N}{\partial \xi}=G, \\
\frac{\partial B}{\partial \xi}=\frac{M_{A}}{\gamma} N Y, \\
\frac{\partial Y}{\partial \xi}=\frac{\sigma M_{i}}{\alpha M_{A}} \frac{G}{N^{2}}-\frac{M_{i}}{\sigma \alpha}\left[\left(1+\frac{T_{i}}{T_{e}}\right) \frac{M_{A}}{\gamma} \frac{C_{s}^{2}}{V_{A}^{2}} G+B N Y\right],
\end{gathered}
$$




$$
\frac{\partial G}{\partial \xi}=\frac{G^{2}}{N}-\frac{M_{i}}{\alpha} \frac{V_{A}^{2}}{C_{s}^{2}}\left(1-\frac{M_{A}^{2}}{\gamma^{2}} N\right) Y N
$$

where $Y=\frac{\partial E_{X}}{\partial \xi}$ and $\sigma=V_{1}-\frac{M_{A}}{\gamma}$. These equations are similar to equations which describe self-organization phenomena in nonlinear dynamics. From these start equations we will calculate the self-organization strucutre, such as shocks and solitons from equations (8)-(11).

\section{INTERMEDIATE SHOCK}

In (8)-(11) the Alfven Mach number $M_{A}$, the ion cycloron Mach number $M_{i}$, the propagating angle $\gamma=\cos \theta$, the initial disturbed velocity $V_{1}$ and the character speed ratio $C_{s}^{2} / V_{A}^{2}$ are parameters. The equations (8)-(11) have various stationary solutions for different parameters combinations. Because the independent variable $\xi$ is travelling wave phase, various stationary solutions are the self-organized structures on the $\xi$. According to the boundary condition $\xi \rightarrow-\infty,(N, B, Y, G)=(1,-\Delta, 0,0)$ we can solve the density, velocity and electromagnetic field of the stable structures from equations (8)-(11).

Figure 1 shows the calculated result of intermediate shock. The solitons series remain in the front of shocks because of no strong dissipation. The angle between the normal of shock and magnetic field is very small, $\gamma=\cos \theta=0.99$. The shock almost propagates along the magnetic field. Figure 1a shows the compressive jump of density. Figure $1 \mathrm{c}$ is the variation of transverse velocity in the shock layer and Fig. $1 \mathrm{~b}$ is the longitudinal velocity. The tangential component of magnetic field is at the upstream and the down stream of shock reverse $180^{\circ}$ (Fig. 1f). As is the electric field.(Fig. le). Compared with MHD shock, there is longitudinal electric field in the front of the shock, which changes along with the solitons on the peak of shock (Fig. 1d). The Alfven Mach number at the upstream of shock is more than 1 (1.54) in the frame of the wave front, and at the downstream is less than $1(0.84)$. There are some solitons remaining in front of the shocks because the kinetic Alfven wave has Landau damping and weak longitudinal electric field dissipation, all of which is matches the expection of the theory.

The calculations show that the existence of intermediate shock depends on the parameters and initial values: (1) Intermediate shock exists only when the angle between the normal of shock and magnetic field is very small. For example, there is no intermediate shock when $\gamma<0.8$. (2) Intermediate shock exists only when $V_{1}-M_{A} / \gamma>0$. This means that speed at upstream of shock should be more than $M_{A}$. The intensity of shock (by the jump of density) sensitively depends on two parameters, the initial disturbed velocity $V_{1}$ and the character speed ratio $C_{s}^{2} / V_{A}^{2}$. The shock is stronger when $V_{1}$ is bigger or $C_{s}^{2} / V_{A}^{2}$ is smaller.

We can roughly estimate the thickness of the shock: $\xi=\left(\alpha X+\gamma Z-M_{A} \tau\right) / M_{i}, \alpha \rightarrow 0$, for the stationary structure, $\xi \sim \frac{\gamma}{M_{i}} Z$ we have obtained $\xi \sim 0.01-0.1$ in calculation, so 


\section{Captions:}

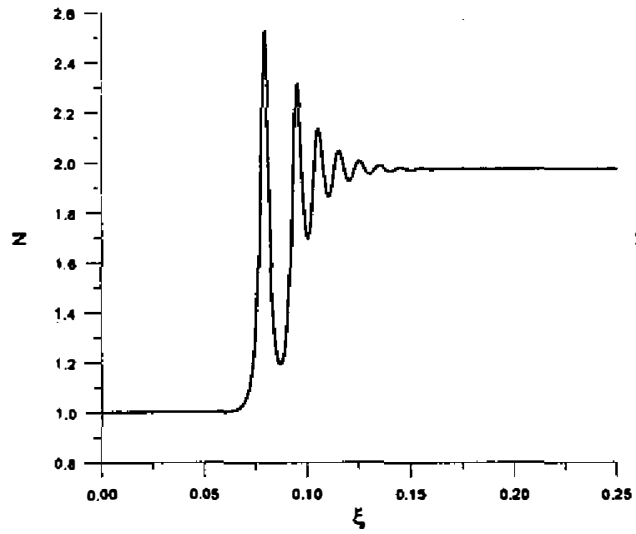

(a)

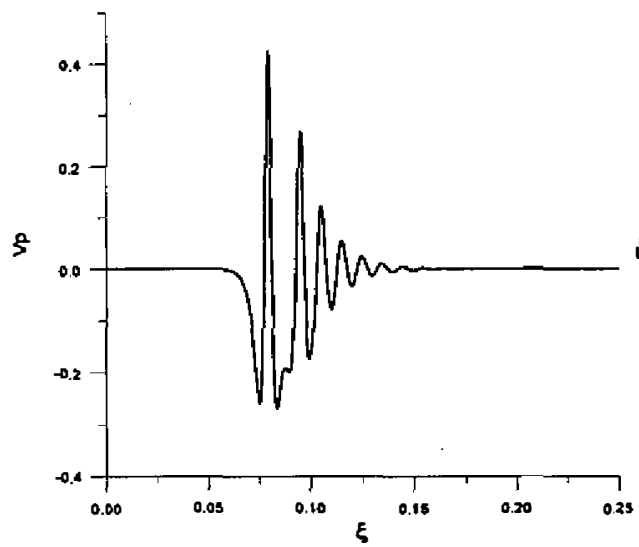

(c)

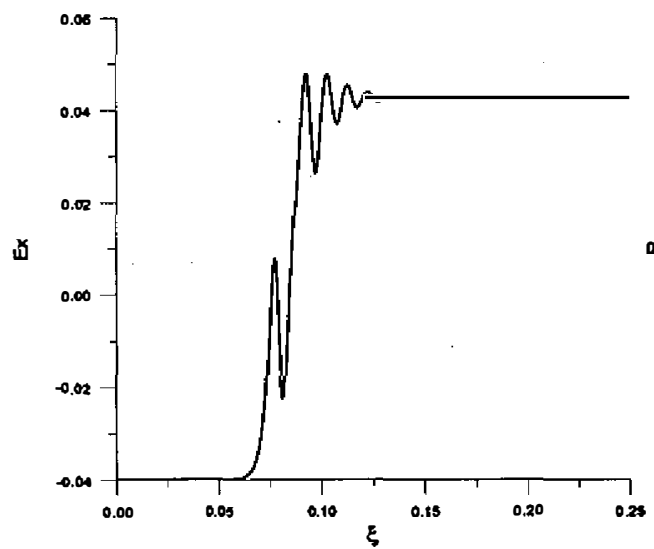

(e)

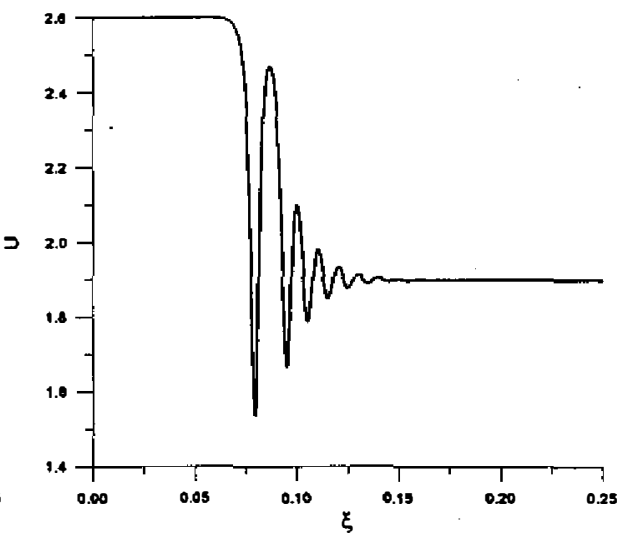

(b)

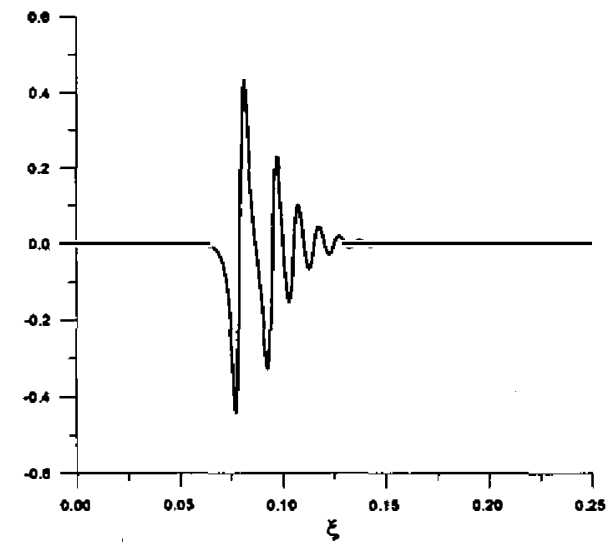

(d)

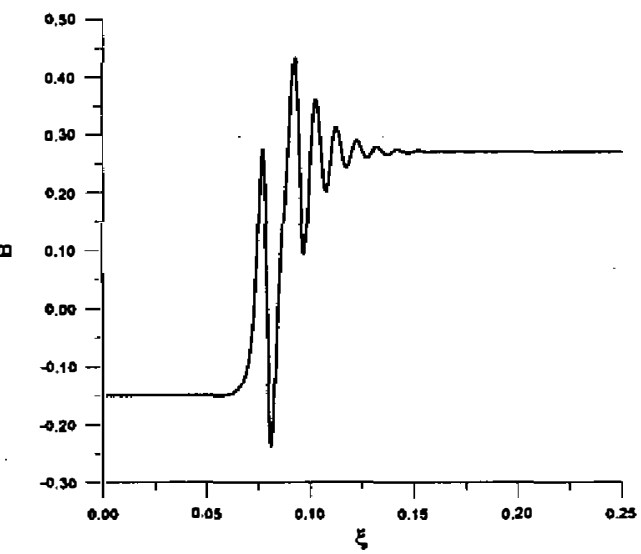

(f)

Fig. I. The solution of intermediate shock calculated from equations (8)-(11) with parameters: $V_{1}=2.6, \gamma=0.9, M_{A}=1.06, M_{i}=200, T_{i} / T_{e}=1, C_{s}^{2} / V_{A}^{2}=$ 0.5 . 
$z \sim \frac{\xi M_{i}}{\gamma}\left(\frac{V_{A}}{\Omega_{i}}\right)=\frac{\xi M_{i}}{\gamma} \frac{V_{A}}{C_{i}} \rho_{i}$. If $C_{s}^{2} / V_{A}^{2} \sim 0.5, \gamma \sim 1, M_{i} \sim 200-1000$, then $z \sim(10-100) \rho_{i}$. We can conclude that the shock is thicker when $C_{s}^{2} / V_{A}^{2}$ is smaller.

Figure 2 shows another example of the calculated result of switch-on shock. The value of parameters has listed in the figure. This switch-on shock evolves from the kinetic Alfven wave propagating toward the sun in the solar wind frame, but carried out by the solar wind. The speed at downstream of shock is smaller than it is at upstream, but it is still larger than the Alfven speed. This seems like a fast shock in the MHD discontinuity theory. But the tangential component of magnetic field of this shock reverses $90^{\circ}$. This result might not have been thought to be an intermediate shock in previous observations. The calculated result indicates that we can find this kind of stationary solution whose velocity at downstream of the shock is still larger than the Alfven velocity. We do not know if anyone has observed such discontinuity in the solar wind. We sort it into switch-on shock. In the shocks mentioned above, the magnetic field at downstream is larger than it is at upstream (Ib type). We did not find the switch-off shock. All such shocks are formed by the compressive solitary wave, with asymmetry $E_{z}$. It is interesting that the rare density at solitary wave (cavitons) can evolve into a rare discontinuity (Fig. 3). Such rare discontinuity also is a type of the stationary solutions of the equations.

\section{ALFVEN SOLITON AND MAGNETIC FIELD KINK}

In various stationary solutions of nonlinear equations (8)-(11), besides the intermediate shocks, there are other important stationary structures, the Alfven solitons and the magnetic field kinks. The existence of Alfven soliton has been proved by Hasegawa (1976) with double electric potential.(Hasegawa and Mima 1976). Figure 4 shows the Alfven soliton solved from (8)-(11). The density is the series of solitary waves (Fig. 4a ). The speeds at upstream and down stream are the same (Fig. 4b). The tangential component of magnetic and electric field rotate $180^{\circ}\left(B_{2 t} / B_{1 t}=-1\right)$ (Figs. $\left.4 \mathrm{e}, 4 \mathrm{f}\right)$. We have tried to find out Alfven shock in various parameters; but we did not find the shock with $B_{2 t} / B_{1 t}=-1$, and $V_{1}>U_{2}$.

In the solutions of Alfven solitons, when the initial impact $V_{1}$ is small, we can find a kind of magnetic kink without the density variation and only the tangential component of magnetic and electric field reverse $180^{\circ}\left(B_{2 t} / B_{1 t}=-1\right)$. This magnetic kink is different from the MHD rotating discontinuit y, just just an Alfven soliton, it propagates with a velocity higher than the Alfven velocity.

\section{INTERMEDIATE SHOCK WITH DISSPATION}

Until now, we considered the intermediate shock without dissipation, that means that in the collisionless plasma intermediate shock (a stationary structure of the epuation) can be formed by the kinetic Alfven wave in the isothermal condition. For MHD shock, intermediate shock without dissipation is impossible; the only condition required for MHD shock is that the entropy increase as fluid passes through the shock. It is not clear what kind of anomalous 


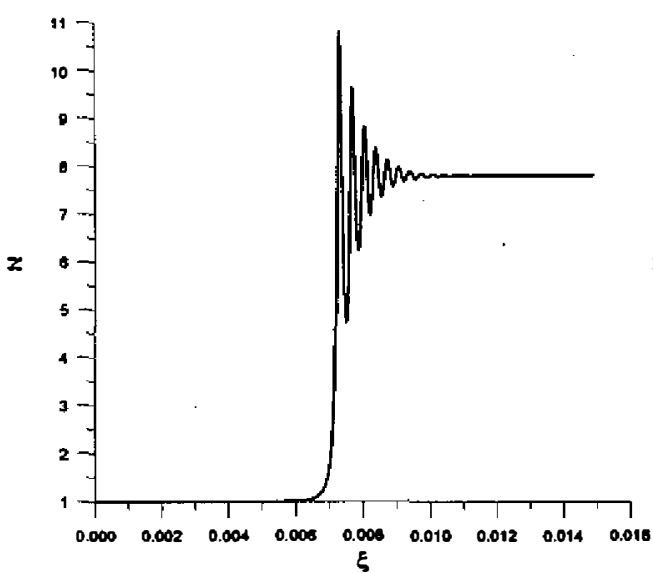

(a)

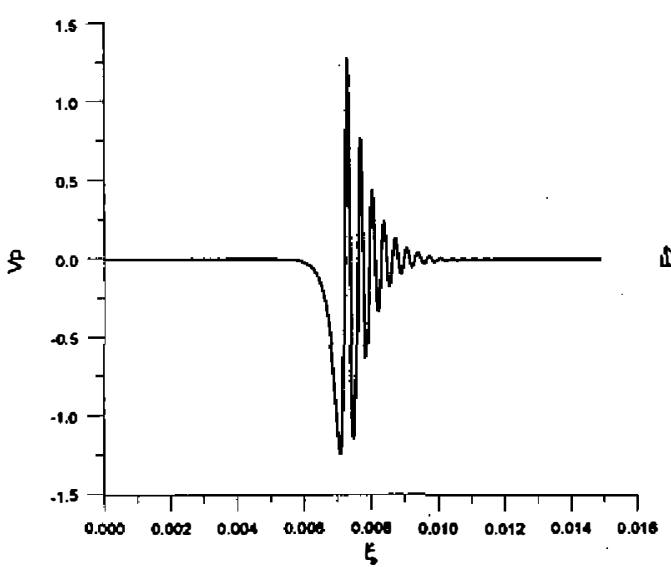

(c)

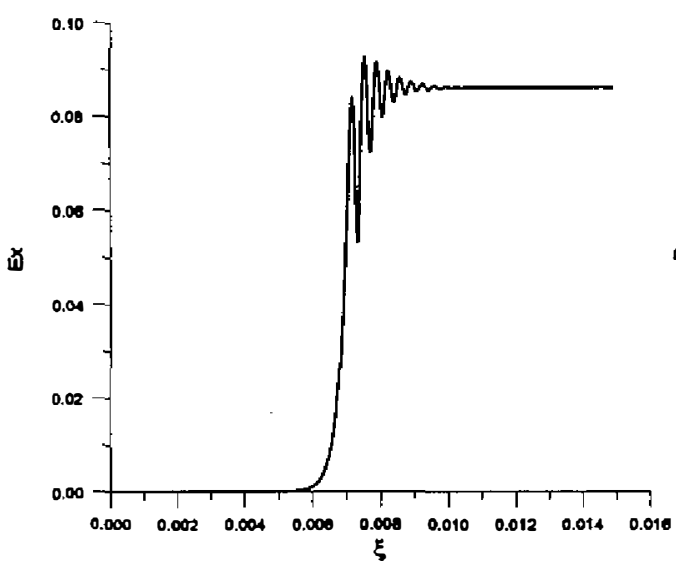

(e)

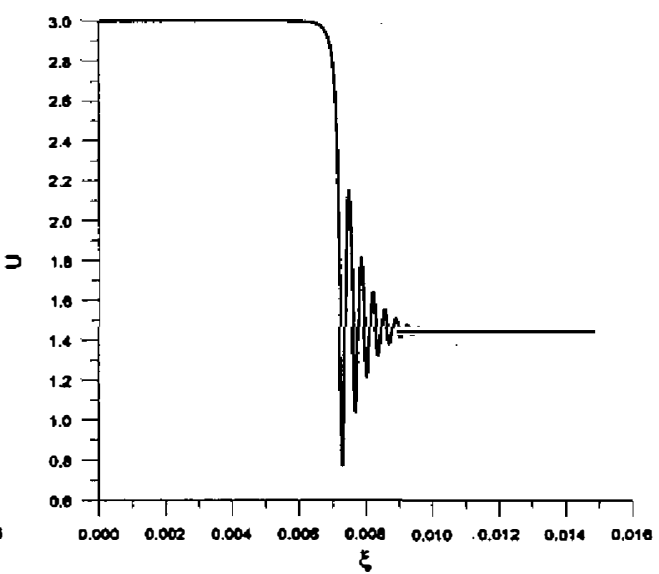

(b)

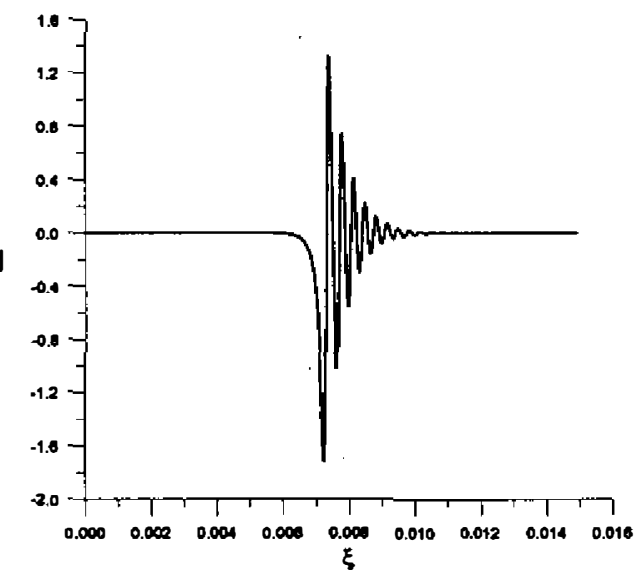

(d)

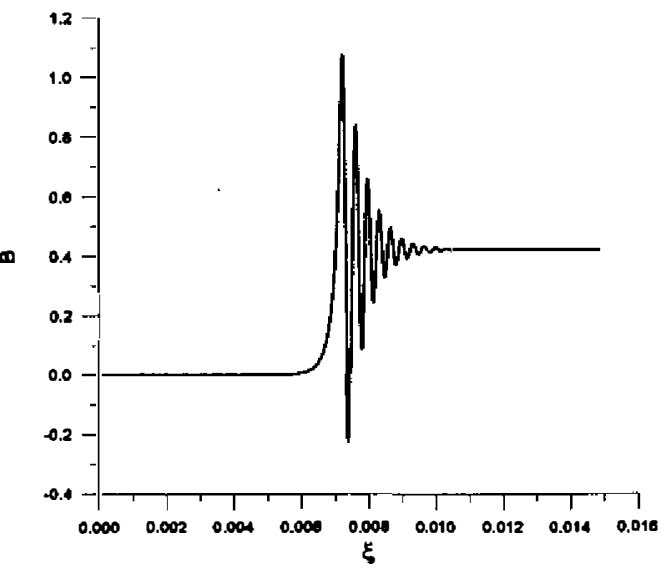

(f)

Fig. 2. The solution of switch-on shock calculated from equations (8)-(11) with parameters: $V_{1}=1.7, \gamma=-0.9, M_{A} \approx-1, M_{i}=100, T_{i} / T_{e}=1, C_{s}^{2} / V_{A}^{2}=0.1$. 


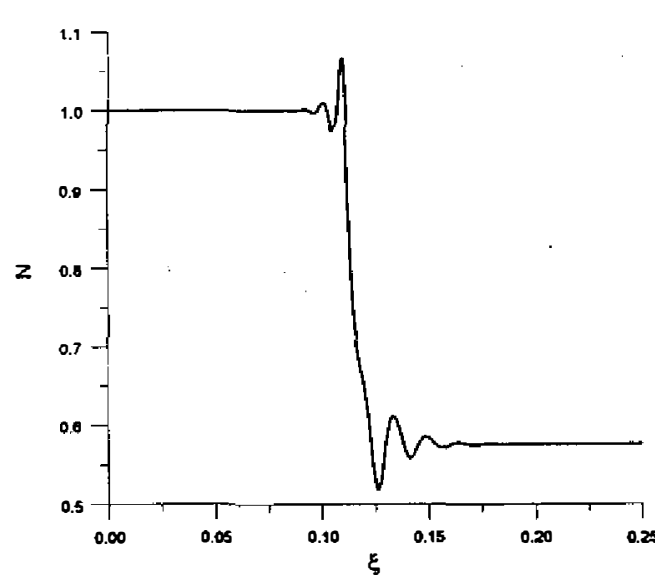

(a)

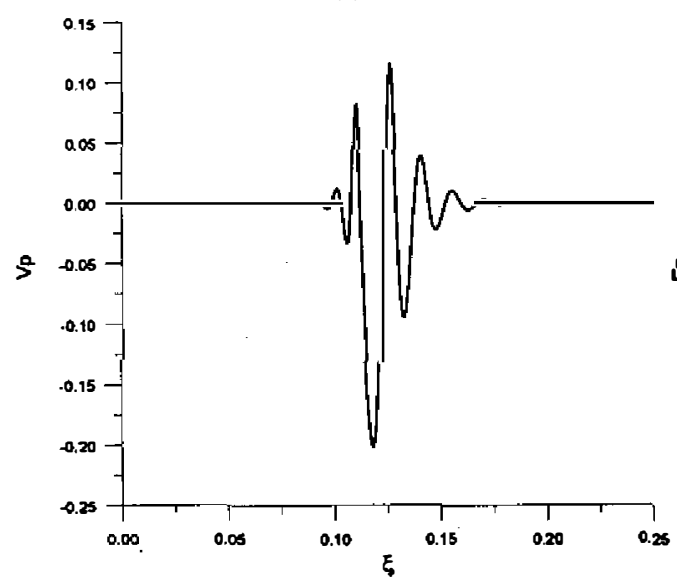

(c)

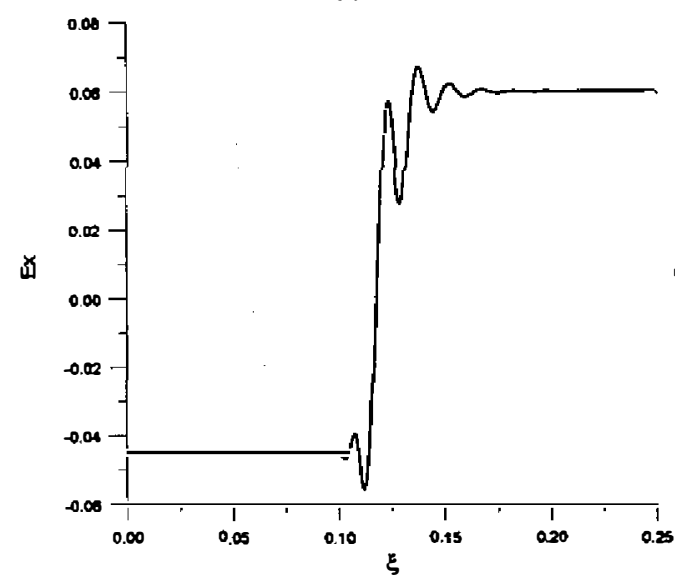

(e)

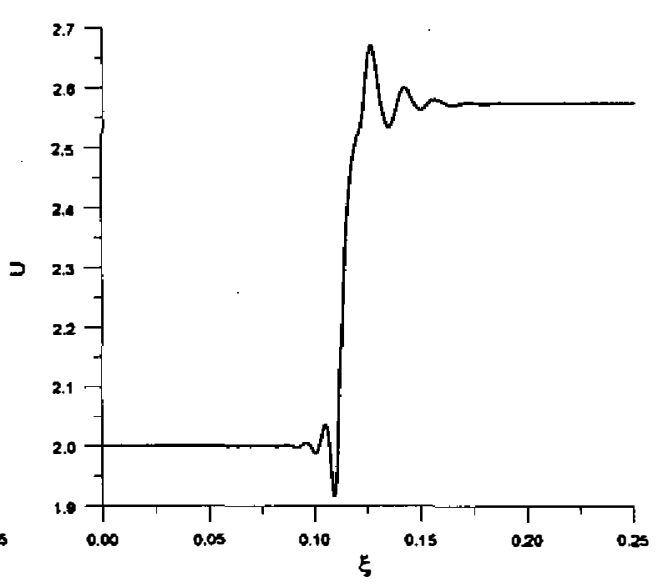

(b)

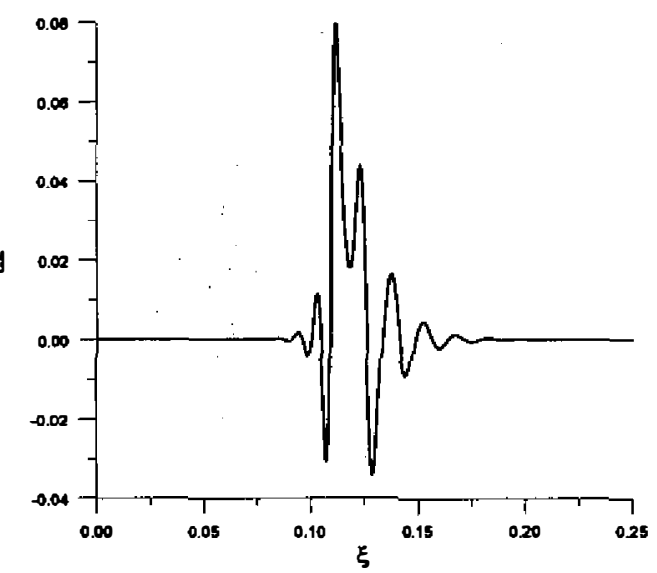

(d)

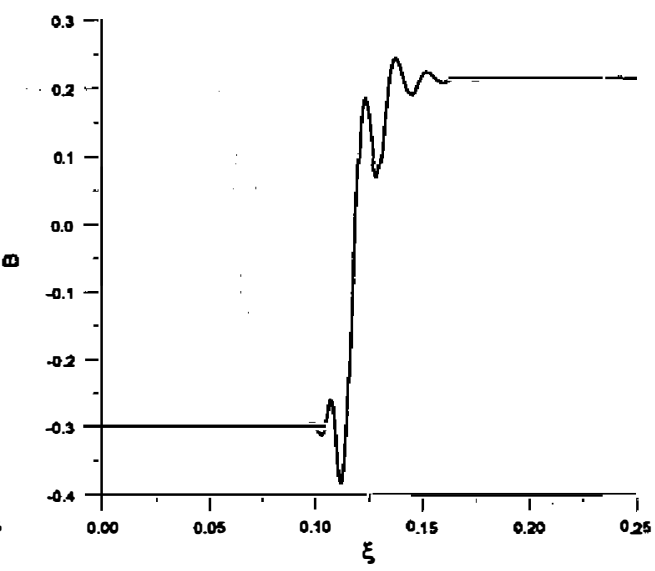

(f)

Fig. 3. The rare discontinuity calculated from equations (8)-(11) with parameters: $V_{1}=2.0, \gamma=0.9, M_{A}=1.1, M_{i}=500, T_{i} / T_{e}=1, C_{s}^{2} / V_{A}^{2}=0.5$. 


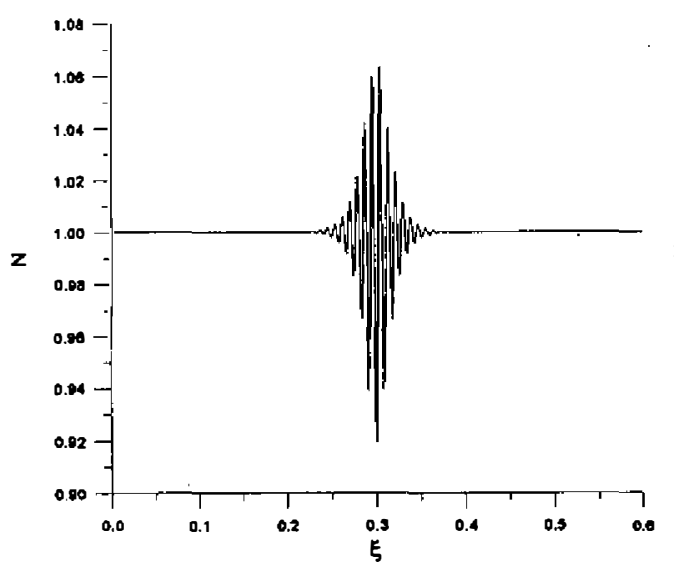

(a)

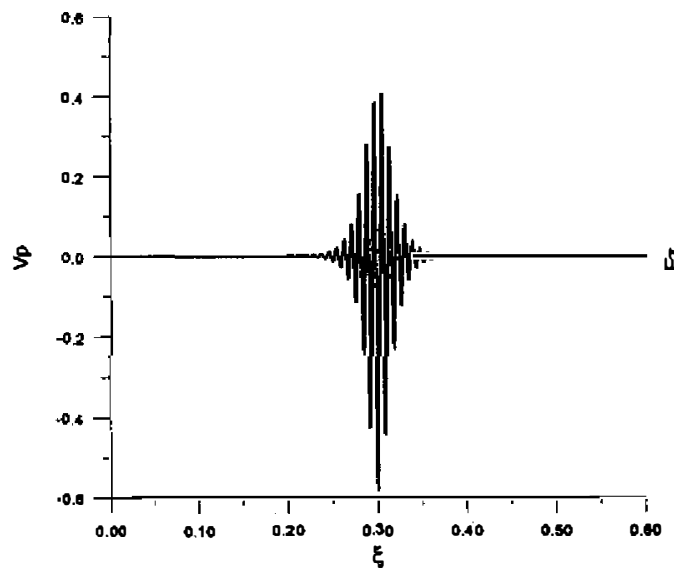

(c)

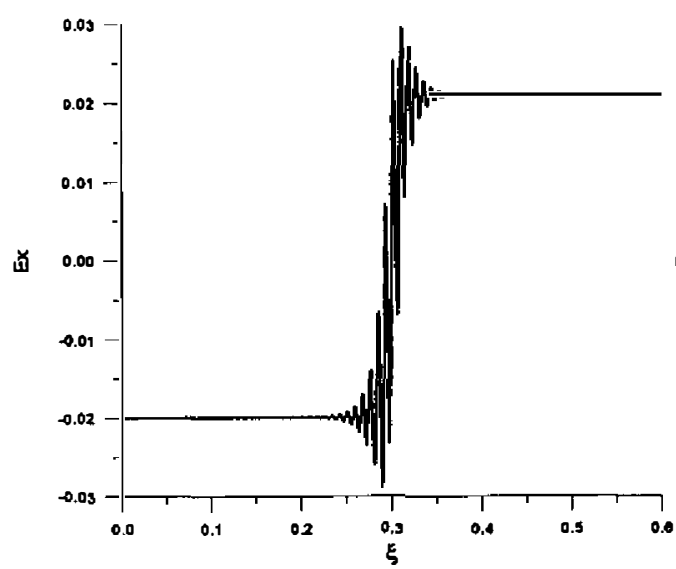

(e)

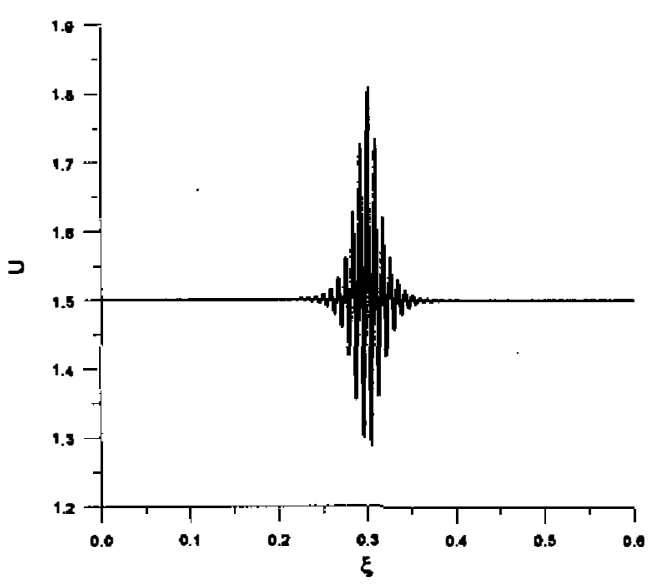

(b)

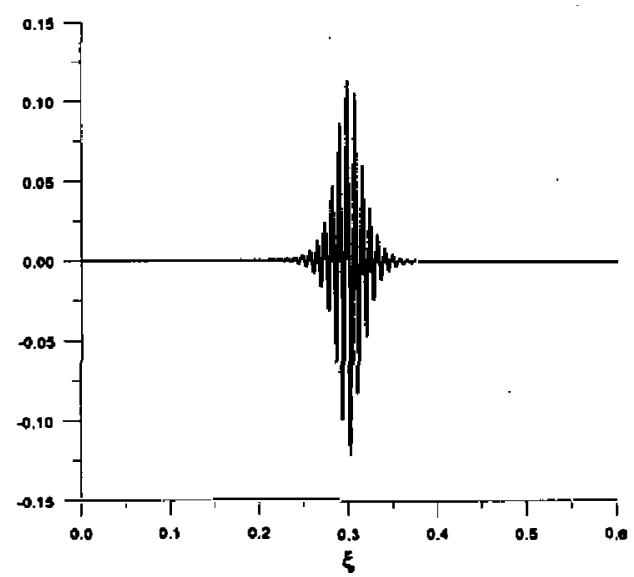

(d)

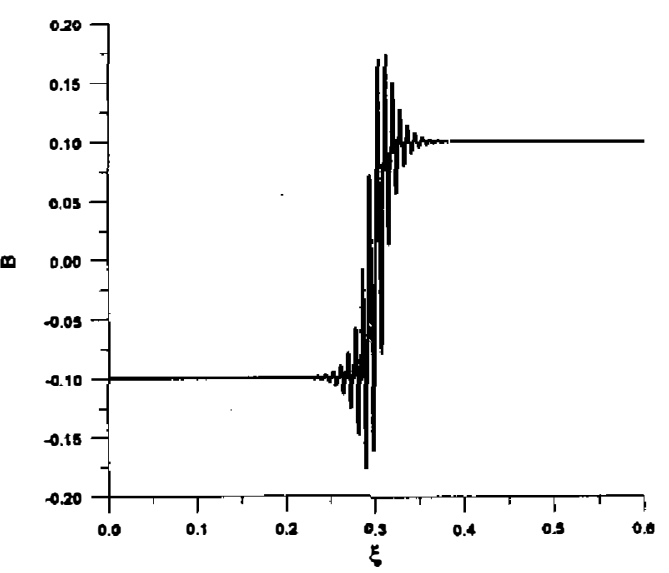

(f)

Fig. 4. The Alfven soliton wave calculated from equations (8)-(11) with parameters: $V_{1}=1.5, \gamma=0.9, M_{A}=1.06, M_{i}=200, T_{i} / T_{e}=1, C_{s}^{2} / V_{A}^{2}=0.5$. 
dissipation exists in the kinetic Alfven waves. Anomalous dissipation of kinetic Alfven turbulence, anomalous dissipation of ion-acoustic turbulence, resistivity of longitudinal electric field, thermal conductivity for collisionless plasma and so on, are possible choice. If some kind of anomalous dissipation in the kinetic Alfven waves is included in the equations, we accept that intermediate shock with entropy increase can be formed. For test we introduce the resistance of the longitudinal electric field and thermal conduction into the energy equation, the equations (8)-(11) became

$$
\begin{gathered}
\frac{\partial N}{\partial \xi}=G \\
\frac{\partial B}{\partial \xi}=\frac{M_{A}}{\gamma}-N Y, \\
\frac{\partial Y}{\partial \xi}=\frac{\sigma M_{i}}{\alpha M_{A}} \frac{G}{N^{2}}-\frac{2 \gamma}{\sigma \alpha M_{A}} \frac{C_{s}^{2}}{V_{A}^{2}}[N F+T G]-\frac{N B Y}{\alpha \sigma}, \\
\frac{\partial G}{\partial \xi}=\frac{G^{2}}{N}-\frac{F G}{T}-\frac{1}{\alpha} \frac{N Y}{T} \frac{V_{A}^{2}}{C_{s}^{2}}\left(1-\frac{M_{A}^{2}}{\gamma^{2}} N\right) \\
-\frac{N}{T}\left(\frac{\gamma \sigma F}{k_{1}}-\epsilon_{1} k_{1}\left(\frac{\gamma \sigma}{N} G T-N M_{A}\left(1+\frac{\gamma \sigma}{M_{A} N}+\sigma Y\right)\left(F+\frac{T G}{N}\right)\right)\right), \\
\frac{\partial F}{\partial \xi}=\frac{\gamma \sigma F}{k_{1}}-\epsilon_{1} k_{1}\left(\frac{\gamma \sigma T G}{N}-M_{A} N\left(1+\frac{\gamma \sigma}{M_{A} N}+\alpha Y\right)\left(F+\frac{T G}{N}\right)\right),
\end{gathered}
$$

where $Y=\frac{\partial E_{X}}{\partial \xi}, F=\frac{\partial T}{\partial \xi} . \epsilon_{1}=\frac{k}{C_{V} m_{i}}$, and $k_{1}=k\left(\frac{\Omega_{i}}{C_{V} B_{0}^{2}}\right) . C_{V}$ is the specific heat of plasma. $k_{1}$ is the thermal conductivity. Here we have omitted tedious derivation. We add the equation (15) which comes from the energy equation and determines the temperature variation as fluid passes through the shock. The parameter $M_{i}$ disappears in equations (12)-(16), because it has been drawn in $\xi$ for simplification. Figure 5 shows the results of calculation. We can see in Fig. $5 \mathrm{f}$ that the temperature increases as fluid passes through the shock, other parameters changes like that in the intermediate shock in Fig. 3. This is intermediate shock with entropy increase as usually understood.

Nonlinear dynamics has an intrinsic property that the solutions of a dynamic system depending on the parameters display complicated behavior: traveling stationary solution appears for some parameters and chaos or turbulence for other parameters. Among so many parameters $\gamma=\cos \theta$, where $\theta$ is propagating angle only when $\gamma \geq \gamma_{c}$ (i.e., $\theta \leq \theta_{c}$ ) the stationary solutions or shocks exist. This is just the requirement of the kinetic Alfven wave. Intermediate shock exists for Alfven Mach number $M_{A}>1$, otherwise the solitary wave or magnetic kink 
(a)
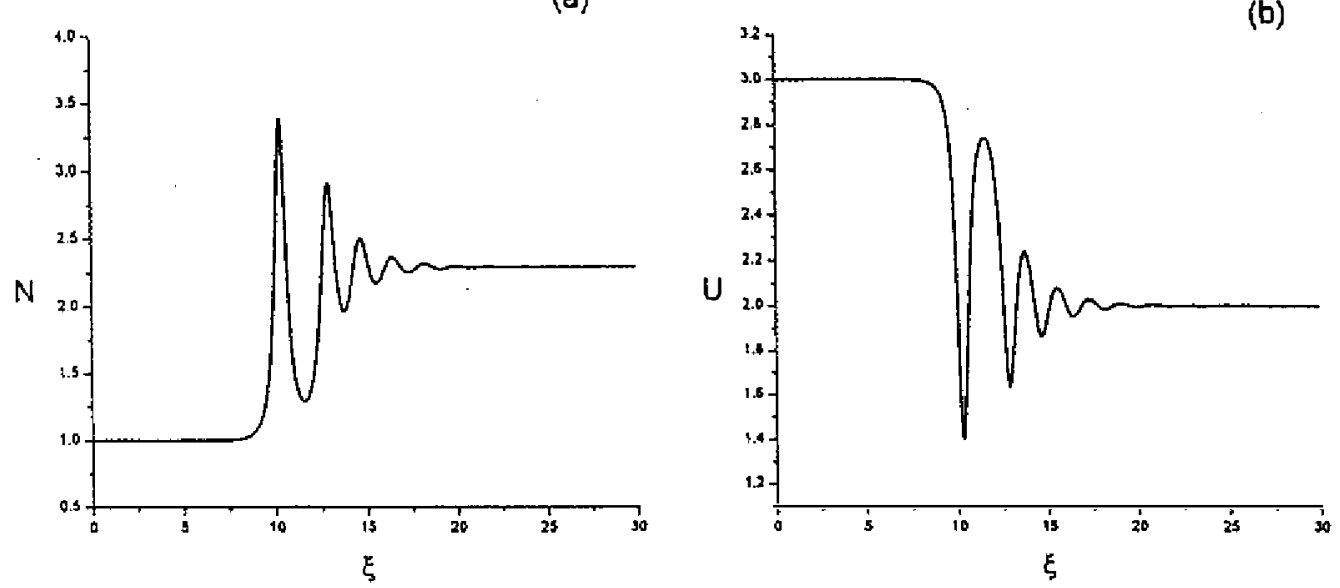

(c)

(d)
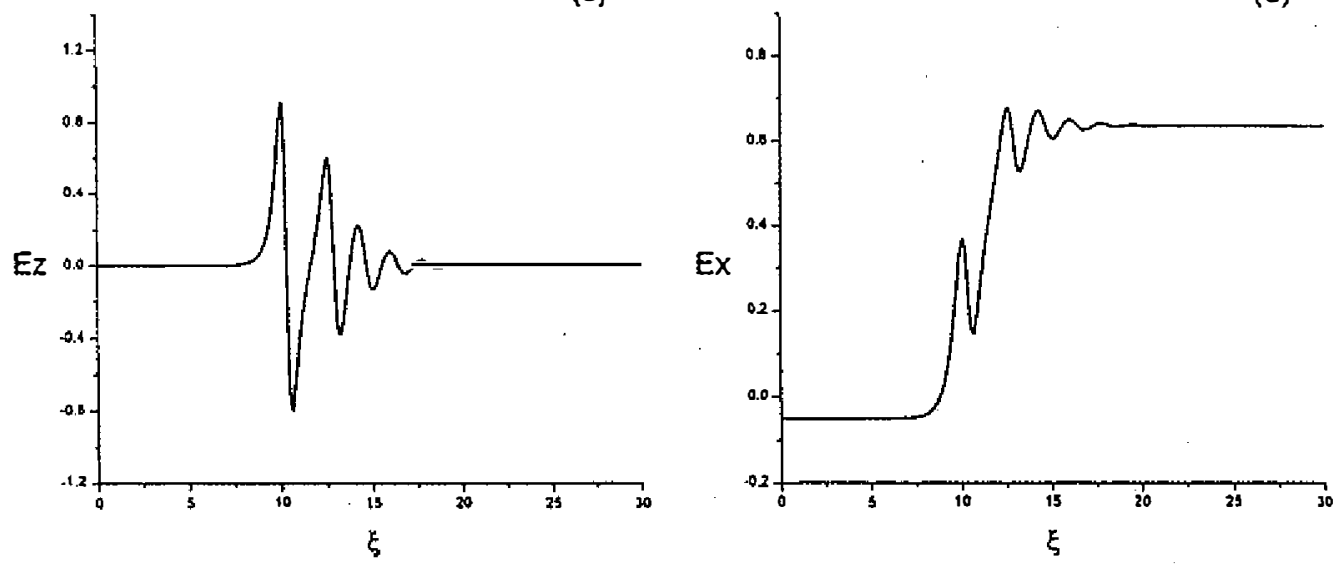

(e)
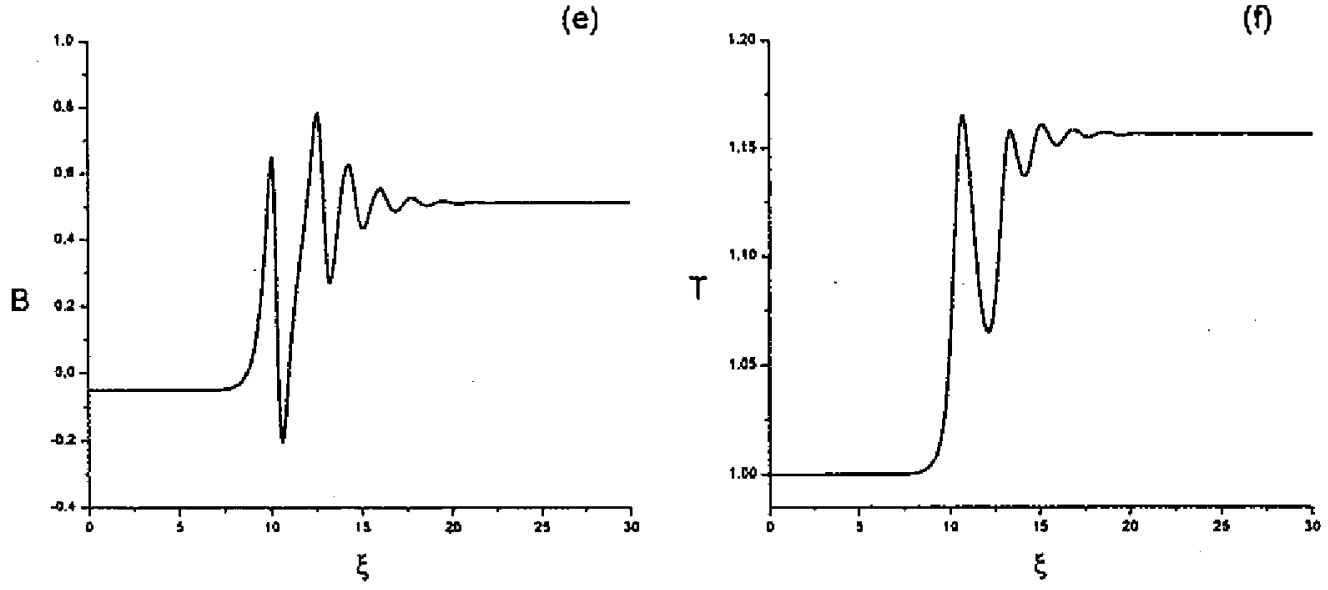

Fig. 5. The solution of intermediate shock calculated from equations (12)-(16) with parameters: $V_{1}=3.0, \gamma=-0.9, M_{A}=-1.1, T_{i} / T_{e}=1, C_{s}^{2} / V_{A}^{2}=0.5, k_{\mathrm{l}}=$ $1.0, \epsilon_{1}=0.055$. 
will be produced The most important parameter is $\sigma$ which is decided by initial impact $V_{1}\left(\sigma=V_{1}-\frac{M_{A}}{\gamma}\right)$. When $V_{1}$ is greater than a critical value the shocks appear; the solitons or magnetic kink corresponding to smaller initial impact $V_{1}$.

\section{CONCLUTION AND DISCUSSION}

The shear Alfven wave with the longitudinal component of electric field and velocity is a coupling mode of the Alfven wave with ion acoustic wave. This wave mode, the called kinetic Alfven wave in nature, is different from the MHD Alfven wave. Hasegawa and Mima (1978) have pointed out that the kinetic Alfven wave is easily exited and develops into the turbulence. The Alfven turbulence in the solar wind perhaps comes from the kinetic Alfven wave, not from MHD turbulence. The kinetic Alfven wave has nonlinear effect, dispersion and even weak dissipation, it easily evolves into a soliton or shock by nonlinear wave form steepening. Transforming independent variable of the dynamic equations into the travelling phase, we derive the nonlinear autonomous equations whose stationary solution is a self-organized structure. This structure can be soliton, shock or something else. If we define shock as a propagating discontinuity, it can exist in the conservative system, but shock with entropy increase can only exist in the dissipated system. Here we have obtained following shocks or solitons by numerical calculation.

(1) intermediate shock (Ib type)

(2) Switch-on shock

(3) Alfven soliton

(4) Rare discontinuity

The method we used to find the solitons can also be used to find the stationary travelling solution of the nonlinear dynamic equations. These equations include the kinetic Alfven wave mode, and allow us to successfully obtained the intermediate shocks. At the front of the shock there are remains of the solitary wave series. The reason is that the kinetic Alfven wave has dispersion and lacks dissipation. We believe that if we add strong dissipation to the dynamic equaitons, such as ion acoustic anomalous resistance, the solitary wave series in front of the shock will dampen off and an intermediate shock with temperature increase will be produced. In fact, we have got intermediate shock with entropy increase (Fig. 5 ) by Joule dissipation of longitudinal electric field.

As the start equations for calculation, in equation (8)-(11), initial impact $V_{1}$ is a parameter. When $V_{1}$ is small, the soliton appears, when $V_{1}$ increases to a critical value, the shock is produced. In the initial equilibrium state $\mathrm{N}=1, \mathrm{Y}=0, \mathrm{G}=0$, but $\mathrm{B}$ (tangential magnetic field) is uncertain; when $\mathrm{B}=0$ we get switch-on shock, when $\mathrm{B}<0$ we get the intermedite shock or soliton. In collisionless plasma, the formation of shock is related to solitary wave, compressive solitary wave corresponds to shock, and the rare solitary wave evolves into a rare discontinuity (Fig. 3).

Once the kinetic Alfven wave is exited it easily evolves into the turbulence. So, the intermediate shock which is produced by the kinetic Alfven wave should be found where the ki- 
netic Alfven wave is excited. At Alfven point of the solar wind (the speed of the solar wind is equal to the Alfven velocity, $U_{0}=V_{A}$ ) there is kinetic exiting of the kinetic Alfven wave ( Song and Lu 1998 ). The new exited kinetic Alfven wave is propagating in two directions along the magnetic field. A branch of the kinetic Alfven wave propagating toward the sun $\left(M_{A}<-1\right)$ is carried by the solar wind outward from the sun. It is mere conjecture that this branch of the kinetic Alfven wave will evolve more easily into the intermediate shock with impact $V_{1}$. Thus kind of intermediate shock can not propagate at $s$ distance. We expect that intermediate shock in the solar wind should exist within $0.2 \mathrm{AU}$. This may be a reason for the lack of intermediate shock in interplanetary space by observations.

Acknowledgment This research was supported by NSFC 49774249.

\section{REFERENCES}

Anderson, T. E., 1963: Magnethydrodynamic Shock Wave, MIT press, Cambridge, Mass.

Chao, J. K., and S. Olbert, 1974: Observasion of slow shocks in the solar wind. J. Geophys., Res., 75, 6394-6397.

Chao, J. K., L. H. Lyu, B. H. Wu, et al., 1993: Observasions of an intermediate shock in interplanetary space. J. Geophys., Res., 98, 17443-17450.

Hasegawa, A., and K. Mina, 1976: Exact solitary Alfven wave. Phys., Rev., Lett., 37, 690.

Hasegawa, A., and K. Mina, 1978: Anomalous transport produced by kinetic Alfven wave turbulence. J. Geophys. Res., 83, 1117-1123.

Hu, Y. Q., 1994: Interplanetary intermediate shocks. ACTC Geophysca Sinia , 34, 397-403.

Jeffrey, A., and T. Taniuti ,1964: Nonlinear wave propagation, Academic,1964.

Kennel, C. F.,R. D. Blandford, and P. Poppi, 1989: MHD intermediate shock discontinuities, Part I: Rankine-Hugoniot Conditions. J. plasma phys, 42, 299-319

Lysak, R. L., and W. Lotko, 1996: On the kinetic dispersion relation for shear Alfven wave.J. Geophys., Res., 101, 5085-5094.

Richter, A. K., H. Rosenbaner, F. M. Neubauer, and N. G. Plitisyna, 1985: Solar wind observasions assoiated with a slow farword shock wave at 0.31 AU. J. Geophys., Res., 90, 7581-7586

Song, L. T., 1996: Kinetic Alfven wave damping and power spectrum of the turbulence in the solar wind (a), Chinese. J. Space Sci., 16, 7-16

Song, L. T., and X. Lu, 1998: the kinetic Alfven excitation of Alfven wave and the capture and acceleration of -particle in the solar wind, Fourth Chinese space science symposium, National Central University, Chung-Li, Taiwan

Steiwolfson, R. S., and A. J. Hundhauson, 1990: MHD intermediate shocks in coronal mass ejections. J. Geophys., Res., 95, 6389-6401.

Wu, C. C., 1987: On MHD intermediate shock. Geophys. Res. Lett., 14, 668-671.

Wu, C. C., 1988: The MHD intermediate shock interaction with an intermediate wave: Are intermediate shocks physical ? J. Geophys. Res., 93, 987-990.

Wu, C., C., 1990: Formation, Structure and stability of MHD intermediate shocks. J. Geophys. Res., 95, 8149-8157. 